\title{
The Future of Electron Microscopy
}

\author{
Yimei Zhu and Hermann Dürr
}

Seeing is believing. So goes the old adage, and seen evidence is undoubtedly satisfying in that it can be interpreted readily, though not necessarily always correctly. For centuries, humans have tried continuously to improve their abilities of seeing things, from developing telescopes to observe our solar systems, to microscopes to reveal bacteria and viruses. The 2014 Nobel Prize in Chemistry was awarded to Eric Betzig, Stefan Hell and William Moerner for their pioneering work on super-resolution fluorescence microscopy, overcoming Abbe's diffraction limit in the resolution of conventional light microscopes. It was a breakthrough that enabled ground-breaking discoveries in biological research and also is testimony of the importance of modern microscopy.

In February 2014 the US Department of Energy's Office of Basic Energy Science held a two day workshop that brought together experts from various fields to identify new science that will be enabled by advances in electron microscopy. [1] The workshop focused on studies of ultrafast processes, atomic resolution, sample environments and measuring functionality at nanoscales. This article captures some of the exciting discussions the workshop generated.

\section{$\underline{\text { Seeing with electrons }}$}

Electrons, photons and neutrons are three fundamental probes of condensed matter. They are routinely used to study complementary physical properties of materials. Neutrons interact with nuclei and atomic spins, x-ray photons with electron clouds and electron probes with electrostatic potentials, i.e., positively charged nuclei screened by negatively charged electrons. Compared with $x$-rays and neutrons, one advantage of electrons is their much stronger interaction with matter $\left(10^{5}\right.$ times stronger than elastically scattered $x$-rays due to the coulomb interaction); hence, for a very small probing volume, the interaction generates strong signals, making it far easier to image and detect individual atoms, molecules, and nanoscale objects. The drawback of a strong interaction is the dynamic scattering effect, often complicating data analysis (as compared to the kinematical approach applicable for $\mathrm{x}$-rays). Another unique feature of electrons is that they are charged particles and can be easily focused using electrostatic and magnetic lenses enabling a wide range of probe size, down to sub-angstrom in scale, and versatile operation modes for imaging, diffraction and spectroscopy, with magnifications from a few hundreds to a hundred million times, on a single instrument.

In a microscope, the resolution limit of two separate objects in a lateral plane is, based on Abbe's criterion set more than a century ago, roughly half the wavelength of the probing media it uses. Taking advantage of the short de Broglie wavelength of electrons (shorter by a factor of $\sim 10^{-5}$ for $60 \mathrm{keV}$ electrons compared to optical light, and much smaller than the size of an atom), German engineers Ernst Ruska and Max Knoll constructed in 1931 the first electron microscope with lenses that are analogous to the glass lenses of an optical microscope. Ruska was awarded half of the 1986 Nobel Prize in Physics "...for his fundamental work in electron optics, and for the design of the first electron 
microscope". The other half went to Gerd Binnig and Heinrich Rohrer for their creation of the scanning tunneling microscope which also uses electrons for imaging.

A conventional transmission electron microscope (TEM) consists of an electron source that emits electrons either by thermionic or field emission, an electron acceleration tube, an electron optical lens system, and various detectors (Fig. 1). The lens system has three segments, each with its own role. The condenser lenses are responsible for beam formation and illumination, the objective lenses for imaging, and the projector lenses for altering magnifications. A magnetic lens comprises coils of copper wires with an iron bore and its magnetic field acts as a convex lens, bringing off axis rays back to focus. Under the influence of the field, electrons follow a helical path, spiralling down the column and passing through a very thin sample, usually $100 \mathrm{~nm}$ or less. In the focal plane of the objective lens a diffraction pattern is formed. In the plane approximately conjugate to the sample, an image is obtained. An objective lens typically consists of an upper and a lower polepiece connected by a yoke. The sample sits in the polepiece gap. A strong lens requires a small polepiece gap, usually ranging from 3-5mm (Fig.1, left panel) often limiting the flexibility of sample maneuverability and the implementation of diverse sample environments. A scanning transmission electron microscope (STEM) is a derivative of TEM where a focused electron beam is scanned across the sample in a raster. Electron-sample interaction generates various signals which often can be acquired simultaneously. The signals can be used for imaging and diffraction as well as for chemical analysis, such as via energy dispersive $\mathrm{x}$-ray spectroscopy (EDX) and electron energy-loss spectroscopy (EELS). In EDX, an incident electron ejects an electron in an inner shell of a sample, creating an electron hole. An electron from an outer shell then fills in, and the energy difference between the two shells is then released in the form of an x-ray for elemental and chemical analysis. In contrast, in EELS, a core electron is excited to unoccupied states, leaving a corehole behind, its energy-loss engenders an energy-loss spectrum. Analyzing its fine structure provides information not only on the local density of unoccupied states, but also on angular momentum and the sample's chemical nature. Lower loss energies can access a large range of quasiparticles excitations as shown in Fig. 2.

Since the invention of electron microscopes, the objective of researchers and engineers has been to improve the spatial resolution of electron microscopes. Progress during the decades before the turn of the $21^{\text {st }}$ century was staggering. Resolution improvements down to 1-2 $\AA$ were largely due to better lens designs and improved electron sources, increased mechanical and electrical stability and the use of high accelerating voltages. The true breakthrough, however, was the development of aberration correctors for the image-forming and probe-forming lenses, respectively, for TEMs and STEMs essentially during the past 15 years. Aberration (spherical or chromatic) causes an electron beam to deviate from the ideal paraxial optics therefore blurring the images. As Otto Scherzer discovered more than 50 years ago, spherical- and chromatic- aberrations are unavoidable for round lenses. An aberration corrector, consisting of multipole lenses without rotationally symmetric electromagnetic fields, can correct these aberrations. However, achieving this requires precise alignment of the corrector elements, which became only possible with modern computer and feedback technology. Today, an aberration-corrected TEM and STEM can generate images with $50 \mathrm{pm}$ resolution, with corrections for the probe-forming and the image-forming lens up to $4^{\text {th }}$ or $5^{\text {th }}$ order of aberrations. The probe corrector is typically located 
above the sample while the imaging corrector is below the sample. Two distinct development paths have been pursued; one for TEM with systems of hexapoles combined with quadrupoles for both spherical- and chromatic-aberration (Haider and Rose) and the other for STEM with systems of quadrupoles and octupoles, mainly for spherical aberration (Krivanek). The effect of chromatic aberration is kept small by introducing a monochromator. TEM and STEM are complementary. TEM offers high acquisition-speed while STEM is ideal for atomic-number (Z) sensitive imaging. Aberration correction in TEM increases its resolution, in particular its image contrast (contrast localization), but caution is needed for image interpretation. In STEM, on the other hand, it supports the opening up numerical aperture of the electron beam, thus enabling to form a sub-angstrom probe with very high probe current for atomic imaging and spectroscopy.

The development of aberration-corrected electron microscopes significantly enhanced our capabilities in electron-beam nanoscale-characterization. Since its commercialization at the beginning of the 21 century, there are now hundreds of aberration-corrected TEM/STEM systems operating at 60-300keV worldwide. Sub-angstrom resolution imaging and spectroscopy is no more a commodity only for large institutions, but also available for researchers at universities. For example, today we can examine in detail a heterogeneous interface of strongly correlated electron materials in two-dimension (projected along the sample thickness). Although it may not be a routine practice we have the capability to visualize not only interfacial atom arrangement via high-resolution imaging, including cation displacement associated with local electric-polarization and individual impurity atoms and dopants, but also interfacial electronic states and charge transfer using atomically resolved spectroscopy.

\section{The new horizon}

In spite of the past success story of electron microscopy, the scientific motivation for improving spatial resolution alone as the future direction is not compelling. There is a strong scientific motivation for achieving atomic or molecular resolution measurements of functionality at short time scales and in real environments. For instance, there are scientific opportunities enabled by probing materials in multiple dimensions, i.e. the three spatial dimensions together with the energy and momentum space. Real materials comprise complex three-dimensional arrangements of atoms and defects that directly determine their utility for energy applications. Consequently, future opportunities will be the 3D imaging of defects, dopants, impurities, and vacancies, along with chemically identifying these impurities in the energy domain to correlate their behavior with different states of electrons, orbitals, and spins. Current ultramodern electron microscopy allows us to image individual atoms and acquire single atom spectra in steady states and in vacuum. For future breakthroughs, there is increasing need for atomic-level imaging and spectroscopy, for example, in liquid, gas, during electrochemical deposition, in oxidation and corrosion in-situ and in-operando, as well as under various applied stimuli (Fig. 1, right panel). The desire to ultimately control materials behavior ranging from molecular dynamics processes over emergence in quantum materials to nanoscale structural disorder and electronic phase separation requires pump-probe-based ultrafast techniques that enable access to the natural length and timescales of the involved elementary processes. The development of a new generation of electron microscopes with groundbreaking fs to ps temporal-resolution and capabilities for measuring materials 
functionality and correlating structure across a wide range of length scales from atomic- to nano- and meso-scale will greatly impact the research areas that are the core of many cross-cutting disciplines. The remainder of this article is therefore sectioned to highlight these aspects.

\section{Seeing materials in multi-dimensions}

Richard Feynman's call "Is there no way to make the electron microscope more powerful?" in his 1959 lecture "There's plenty of room at the bottom" had a significant impact on the development of aberration corrected electron microscopes along with the worldwide nanoscience initiatives. However, a challenge extending beyond Feynman's vision to understand the quantum world of materials is to develop atomic-resolution multidimensional microscopes to locate and identify all atomic species in complex systems. The term multidimension here refers to three-dimensional tomography and spectroscopy of atomic- and electronic-structures over real space, momentum space, energy space, time space, and additional space or dimensions through various external excitations (see Fig. 2). The properties of functional and quantum materials are determined by collective and coupled interactions, such as strong Coulomb interactions among the electrons, strong electron-phonon coupling, polarons, spin, charge, and orbital ordering and fluctuations. These interactions are at the heart of some of the deepest unsolved mysteries in the physics of condensed matter and engender extraordinary phenomena such as high-temperature superconductivity. Very often, these intriguing properties result from nanoscale electronic inhomogeneity, broken symmetry, or phase separation. Understanding, controlling, and manipulating these local phenomena is of great importance for next-generation information and energy technologies. It requires imaging the real-space atomic structure, electronicand spin-structure in multidimensions.

The rapid development on electron tomography, which is benefiting from the introduction of aberration-corrected electron optics, led to the visualization and analysis of three-dimensional structural- and chemical- information from materials at nanoscales. It was demonstrated that, with novel reconstruction algorithms, not only imaging atoms in 3D, but mapping electrostatic and magnetic potentials in practical $p-n$ junctions and devices is possible if combined with Off-axis electron holography. One main challenge and opportunity for future 3D imaging is to resolve and identify individual impurity atoms in both periodic and aperiodic structures of materials including amorphous compounds that consist of different chemical species. 3D imaging entails acquiring a series of images, thus imposing a significantly higher electron dose on the samples and radiation damage will become an increasingly important issue. Low-energy electrons under low-dose operation may be one of the solutions for future instrumentation development.

Electron energy-loss spectroscopy (EELS) in TEM and STEM offers the possibility of probing materials in energy space and in momentum space, exceeding the capability of EDX which is mainly used for composition analysis. The angular momentum of the final state of a sample is determined by the angular momentum of the initial one, and the electric-dipole approximation and selection rules. When a sufficient small probe is formed to excite electrons only on a single atom or atomic column, site specificity information on the core-level binding energy can be obtained. The analyses of the EELS near- 
edge fine structure, in particular at low temperatures where many interesting phenomena in condensed matter occur, not only enable us to gain information on electronic states but also on spin- and orbitalmoments. The low-energy-loss regime provides information on plasmonic excitation, electron-phonon coupling, lattice vibration, and phonon density of states as shown in the taxonomy of excitations in Fig. 2. Currently most of the low-energy excitations shown are not accessible to EELS due to the limited energy resolution, ranging from $0.7 \mathrm{eV}$ and $0.3 \mathrm{eV}$ for thermionic and cold field-emission gun, respectively, to $0.1 \mathrm{eV}$ and below for monochromated instruments. With an energy resolution of a few meV [2] both optical and acoustic phonon modes as well as magnon and many-body absorptions can be observed. This new capability will allow us to directly correlate the structure of interfaces, defects, inclusions, impurities (e.g., a "rattler" ion), and local bonding to the subtle changes of the phonon spectra, and garner knowledge that is very relevant to a wide range of frontiers, such as phonon scattering, heat transport and electron correlation. Another benefit of the electron probes is its access to a much large momentum space, compared with x-rays, due to its shot wave length or flat Ewald Sphere that intersects with reciprocal lattices. A new direction for EELS will be the angular resolved measurement, i.e., acquiring EELS data as a function of moment transfers (or q vectors), similar to angular resolved photon emission spectroscopy (ARPES), but on individual atoms in a bulk material. For such capabilities to become a routine reality major instrumentation developments are required, including new ultra-bright electron sources, significantly improved electronic and mechanical stability, revolutionary electron optics designs and ability to operate the instrument at liquid He temperature.

\section{Seeing materials from in situ to operando}

The discovery and design of future materials and devices strongly depends on having a complete understanding of materials's behavior under external stimuli, as illustrated in Fig.1 (right panel). Modern electron microscopy plays an increasing role in characterizing ferromagnetic and ferroelectric nanostructures and their switching behavior in-situ. The need to continuously decrease the size of bitelements in nonvolatile memories perhaps is the main underlying driving force. The switchable spontaneous polarization, domain-engineering and strain-control of ferroelectrics recently were also targeted for ferroelectric field-effect transistors. Currently, Lorentz microscopy with variable applied magnetic fields is widely used to reveal previously unknown spin configurations, such as skyrmions, and their evolution. Capabilities for $\mathrm{GHz}$ resonance and pulsed excitations in-situ have been developed to study spin-toque effects using continuous electron beams in conventional TEM. A local electric or magnetic field or potential can be applied to a nano-metric area in the microscope using a piezo-driven needle built into a sample holder to study magneto electric behavior of a memory device or ionic transport in Li-ion nano-batteries.

Today complex experiments using external stimuli, in particular under samples' working conditions (operando) that require a large sample environment mainly are carried out using $x$-ray and neutronscattering methods, which habitually only provide averaged structure information determined by the beam size. Proximal-probe approaches, including atomic-force microscopy and scanning-tunneling spectroscopy, offer information on the local electronic structure and have revealed electronic 
inhomogeneity and phase separation, but are explicitly surface-and near-surface-sensitive and difficult in applying external stimuli. Electron microscopy is the method of choice to reveal the local atomic-, electronic-, and spin-structures that are critical to understanding the materials' behavior at the nanoscale [3]. It is the only method that allows us to study weak scattering objects, for example, singlewall carbon nanotubes and graphene, using atomic imaging, diffraction and spectroscopy from the same areas of interest, due to the strong interaction of electrons with matter. The advent of spherical- and chromatic-aberration-correction technology has made it possible to widen the gap between the polepiece in electron microscope without compromising much of its spatial resolution. The future needs include a $1 \mathrm{~cm}$ or more polepiece gap. It will provide much required space for designing more sophisticated sample holders for in-situ experiments, enabling imaging and measurements under applied stimuli and even in capsulated forms for gas and liquid, whose development is largely due to the technical advances in nanofabrication- and membrane -technology. The shortcoming of the capsulated cells is the membrane itself, usually $\mathrm{Si}_{3} \mathrm{~N}_{4}$, is in the beam path, which considerably reduces the transmitted and scattered electron signals while increasing the background. As an alternative, open-cell configuration for gas- and liquid- experiments have also been developed, e.g., for liquid using low-vaporpressure ionic liquids, or for gas using differential pumping systems with up to 20 Torr gas pressure in the microscopes. For both approach to achieve seeing materials' behavior in operando major instrumentation development is required.

There are other longstanding challenges and opportunities in in-situ electron microscopy. One of which is to image light atoms/ions and vacancies and their electronic states in gaseous- and liquidenvironments, and imaging even at low magnifications, say sub-micron scale, can pose major problems due to the presence of gas or liquid. High-energy electron beams are ideal for penetrating thick liquid cells that allow liquid to circulate, or gas cells that can inject a gas pressure close to that of the atmosphere. However, they induce more knock-on damage to the samples. Low-energy electrons, on the other hand, interact more strongly with matter (shorter scattering mean-free-path) than highenergy electrons, so they generate better image contrast, but they penetrate much thinner samples, thus low signal and high background. Radiolysis is another radiation damage mechanism which usually does not depend on the energy of electrons. It can be a major issue in the presence of liquid and gas. To detangle the structural dynamics and chemical reactions of the samples under study from artifacts in a gaseous- and liquid-environment we have to understand the complex electron-beam induced effects. Low electron-dose imaging and fast data acquisition, the similar approach used in structural biology, are essential in minimizing unwanted artifacts. Individual instruments have to be built for specialized research direction, like individual beamline in a synchrotron facility, no single instrument can fit all.

Game-changing opportunities: the new and exciting science with ultrafast electron microscopy

The recent development of ultrafast imaging and diffraction opened a new frontier for studying structural dynamics at the nanoscale. It has become one of the key directions for future electron microscopy. Ultrafast electron microscopy combines the superior spatial resolution of conventional electron microscopy with femtosecond short electron pulses enabling the detection of electronic and 
atomic motion on their natural time and lengths scales [4]. While electrons in solids typically travel with the Fermi velocity (typically several $\mathrm{nm} / \mathrm{fs}$ ), atomic propagation occurs with the speed of sound (typically several $\mathrm{nm} / \mathrm{ps}$ ). These simple estimates illustrate the required ultimate temporal resolution for probing atomic and electronic motion and the intrinsic coupling mechanisms between these degrees of freedom. Present day ultrafast electron microscopy instruments achieve the ultimate space-time resolution (nm.ps) in the single electron limit (one or few electrons per pulse, see for example [5]). The single electron per pulse approach circumvents beam deterioration and image blurring that is a significant limitation for multi-electron pulses due to the repulsive electron-electron Coulomb interaction. However, it requires a multimillion-shot accumulation to get a decent image [5]. This is technically only feasible by using instruments that operate at $\mathrm{MHz}$ repetition rates. Such instruments only probe repeatable dynamic events. In addition the sample needs to be reset to the initial conditions before the next electron pulse arrives. Irreversible phenomena require images to be taken by individual electron pulses containing a sufficient number of electrons $\left(\sim 10^{6}\right.$ or even more). Presently single-shot ultrafast electron microscopes, such as the one developed at Lawrence Livermore National Laboratory, have to operate with much longer electron pulses $(\sim \mu \mathrm{s})$ to preserve electron beam quality for achieving $\mathrm{nm}-\mu \mathrm{s}$ resolution (10-100 nm, 10-100 ns). Future instruments that pack more electrons into each pulse while preserving the beam quality and ultrashort pulse length require an increase of the electron beam energy. Among the state-of-the-art electron sources, only photocathode RF guns developed for x-ray free electron lasers (XFEL) such as the Linac Coherent Light Source in Stanford, are capable of generating $>10^{7}$ electrons per pulse, multi-MeV energy and sub ps-short electron beams. The distinct merit of photocathode RF guns is the very high acceleration gradient ( $>100 \mathrm{MV} / \mathrm{m})$, over two-order of magnitude higher than 200KeV DC-guns. It enables us to considerably minimize the electron-electron repulsion effects, which can be scaled as inversely proportional to the product of velocity square and energy cube of the electrons, thus yielding much higher current density from the cathode and significantly improving beam brightness by at least the same amount, as recently demonstrated at Brookhaven National Laboratory. The use of rapid acceleration to $\mathrm{MeV}$ electron beam energy offers sufficient electrons per pulse for single-shot capabilities. A further important advantage of $\mathrm{MeV}$ electron beams is their relativistic propagation speed that essentially eliminates the velocity mismatch between the electromagnetic pump pulses and the probe electrons. These $\mathrm{MeV}$ electron beams also penetrate thicker samples and reduce multiple scattering contributions due to the longer elastic mean-free-path, thus, enabling easier interpretation. There are significant challenges that lie ahead of us in the associated with the development of ultrafast electron microscopes, both through modifications of commercial TEMs and home-built accelerator based instruments. However these challenges are outweighed by the scientific opportunities opened up by achieving unprecedented spatial and temporal resolution In the following we use the complementarity of electrons and XFEL based $x$-rays as a guide to illustrate the fascinating scientific opportunities with this new generation of ultrafast electron microscopy instruments.

Energy transport at the nanoscale is of crucial importance for a wide range of materials [1]. One example that all of us encounter on a daily basis revolves around the heat management in modern microprocessor computer chips where overheating is a significant performance limit. Our ability to tailor heat dissipation in new materials and nanostructures is currently handicapped by detecting and 
understanding energy transport as it occurs on microscopic length scales and across interface boundaries. The experimental challenge is to determine the scattering events leading to changes in local phonon populations during heat transport. Instead of operating in the frequency domain where quasiparticle excitations such as phonons appear as discrete energy-loss features in EELS (see Fig. 2), time-resolved techniques detect the coherent oscillations of phonons as they evolve in time and momentum space. Fig. 3 shows a recent example obtained using femtosecond x-ray pulses [6]. In this approach the atomic oscillating motion caused by excited phonon modes leads to diffuse scattering intensity away from lattice Bragg peaks. The distance from a Bragg diffraction peak in momentum space at which such a diffuse scattering yield oscillation is observed is directly determined by the wavevector of the propagating phonon [6]. Spectroscopic information, i.e. the phonon frequency, is obtained via the Fourier transform of time-resolved coherent oscillations of the diffuse scattering signal. Retaining the phase information in a time-domain measurement provides a unique opportunity for a detailed microscopic understanding of coupling between quasiparticle modes in emergent order and for probing excitations in materials under far-from-equilibrium conditions. Such measurements have just now become possible with XFELs [6] and have been started to be performed at existing ultrafast MeV electron diffraction sources, however in both cases without needed resolution in real space. Ultrafast $\mathrm{MeV}$ electron diffraction offers the advantage that electron scattering cross sections at comparable wavevectors are many orders of magnitude higher than those for x-rays. This is especially important for the inherently low intensity of diffuse scattering signals. However the truly game-changing quality of ultrafast electron microscopy is it being the only technique that can simultaneously achieve fs time and $\mathrm{nm}$ spatial resolutions. A possible future implementation is illustrated in Fig. 3 where the nanoscale focusing of electrons enables the detection of a local diffraction pattern at a spatial resolution given by the $\mathrm{nm}$ electron beam focus. This will for instance allow the direct visualization of nanoscale heat transport and the understanding of phonon scattering mechanisms as they evolve in time and space after localized energy deposition using, for instance, nanoscale plasmonic field localization.

Detecting the coherence of electro-magnetic excitations is of central importance for strongly correlated electron systems. The competition between various degrees of freedom such as spin, orbital, charge and lattice makes these systems very sensitive to external perturbations providing a pathway towards controlling their spectacular materials properties of which high temperature superconductivity is probably the best known. The relevant energy scale for controlling emerging new states is believed to be in the $\mathrm{THz}$ frequency range ultimately being determined by the energy differences (note: $1 \mathrm{THz}$ corresponds to an energy of $4 \mathrm{meV}$ ) of competing ground states. This raises the interesting prospect of controlling materials' properties via $\mathrm{THz}$ excitation. Groundbreaking experiments reported the emergence of transient superconducting behavior out of the charge and spin-ordered insulating state by resonant excitation of $\mathrm{THz}$ lattice vibrations [7]. These reports are provocative and the search for competing phases is currently a hot topic using fs x-ray pulses at XFELs. However, the ground state of high temperature superconductors and quantum materials in general, is well known to be spatially inhomogeneous at the nanoscale. The possibility to access the sample response at the length scale of imperfections with ultrafast electron microscopy combined with tailored photonic excitation can be expected to transform the field. Ultrafast electron microscopy will also allow unambiguous access to lattice excitations that are coupled to the spin and charge degrees of freedom in many other quantum 
materials. Detecting the coherent excitation of quasiparticles such as electro-magnons (coupled electronic and magnetic excitations) in multiferroic materials has very recently been reported using multi-cycle $\mathrm{THz}$ pulses [8]. This permits exploration of quasiparticle interactions and phase transformations induced by strong electric fields in these materials. Strong pulses [8] are expected to be able to switch the electrical polarization state possibly influencing magnetic order in these materials (Fig. 4). Ferroelectric and ferromagnetic heterostructures are expected to enable switching of the ferromagnetic state and offer novel ways to magnetically store information without the need of applying magnetic fields driven by dissipative electrical currents. Static and even dynamical phase separation scenarios are believed to be very common in these materials. Ultrafast electron microscopy will thus enable radically new investigations in space and time of the application potential for data storage devices.

Although for applications the reversible switching of materials properties needs to be explored one has to be able to investigate irreversible processes. Ultrafast electron microscopy offers the game-changing opportunity in this respect. The possibility of packing many electrons into relativistic MeV electron bunches will enable obtaining snapshots down to few ps or even sub-ps long electron pulses opening up the possibility of snapshot imaging on mesoscopic length-scales ranging from nanometers to micrometers. This is expected to significantly extend current-day capabilities (see above). Examples for applications of such a capability range from protein dynamics over advanced materials synthesis to matter far from equilibrium (warm dense matter). Probing materials at ultrahigh temperatures and pressures will not only be critical for the understanding of warm dense matter but also for non-periodic systems such as glasses and highly nonreversible glass transitions. Electron scattering is a natural choice for such systems. With the development of high-quality electron lenses including superconducting ones multiple length scales can be imaged in a single shot. Combining with diffraction and spectroscopy it will allow determining not only the evolution of morphology and composition of a sample but also the dynamics of its atoms, electrons and spins.

\section{Concluding remarks}

In summary, we have discussed the breakthrough science opportunities and the needed instrumentation development for future electron microscopy. It is clear that the world of probing matter with electrons will experience revolutionary growth in capabilities in the next decade from ultrasmall to ultra-fast and to multidimensions. Probing materials' functionalities in-situ and in operando with high spatial- and temporal-resolution will continue to be a main theme. Although enabling instrumentation is often specific for scientific opportunities, there are cross-cutting areas include developing bright electron sources, fast detectors and dedicated spectrometers. As modern science moves from the understanding of structure and ground states to dynamics and functionalities, such developments would result in transformative advances within fields of science or engineering that allow to better address our national and international needs. 


\section{$\underline{\text { References }}$}

[1] http://science.energy.gov/bes/news-and-resources/reports/

[2] O. Krivanek et al, Nature 514, 209 (2014).

[3] MRS Bulletin, Special issue on Frontiers of In Situ Electron Microscopy, H. Zheng, S. Meng, Y. Zhu Eds. Jan. 2015.

[4] M. Trigo et al. Nature Physics 9, 790 (2013).

[5] A. Zewail, Science 328, 9 (2010).

[6] T. Kubacka et al, Science 343, 1333 (2014).

[7] D. Fausti, et al, Science 331, 189 (2011).

[8] D. Daranciang, et al, Appl. Phys. Lett. 99, 141117 (2011).

\section{Figure Captions}

Figure 1: (left) Cross section of a typical transmission electron microscope and detectors assignment (1: energy dispersive x-ray spectroscopy (EDX) detector, 2: back scattered electrons and secondary electrons detector, 3: bi-prism for off-axis electron holography, 4: scanning transmission electron microscopy (STEM) detector, 5: TV camera, and 6: electron energy-loss spectroscopy (EELS) and spectroscopy imaging detector. A schematic of the sample area along with the objective aperture and contamination reducing cold-trap within the pole piece gap of the objective lens is also included. The useable dimension for a sample holder or environment along the optical axis in the gap is usually $3 \mathrm{~mm}$ for an ultrahigh resolution microscope, while $5 \mathrm{~mm}$ for a high-resolution analytical microscope. (right) Schematic of in-situ electron microscopy with a continuous or pulsed beam 3D imaging and spectroscopy over real space, momentum space, energy space, time space, and external excitations including temperature, electromagnetic field, optical pump, mechanical stress and liquid and gas environment. Various signals including energy-dispersive X-ray spectra and electron energy-loss spectra are collected by dedicated detectors to map energy and momentum space and to understand materials' functionalities and behavior.

Figure 2: Exploring momentum space and energy space with electrons. Taxonomy of excitations as they become accessible to energy loss spectroscopy (EELS) in electron microscopes. With increasing in energy resolution in EELS both phonons and electronic excitations within the atomic valence and excitations between core and valence shells are visible. Probing different excitations in momentum (reciprocal) space is indicated by the diffraction pattern. While core-valence excitation shown no chance with crystal momentum, many excitations especially at low energy display changes of the loss energy with momentum that allow mapping on corresponding quasiparticle motion in solids. At intermediate energies, electronic excitations within the d-shell of transition metal (TM) ions (d-d) as well as chargetransfer excitations (CT) and excitations across the gap of Mott insulators display reduced dispersions.

Figure 3: Left: Time-resolved diffraction offers a radically new way to observe quasiparticle motion in solids that is conventionally probed in energy-momentum space (see Fig. 2). The figure shows 
femtosecond x-ray scattering measurements of phonon dynamics in Ge. The diffraction snapshots taken stroboscopically at 0.4 ps, 1.2 ps and 2.8 ps after exciting a Ge single crystal with a 100 fs long optical laser pulse display the relative changes in diffraction intensity as phonon excitations oscillate in space (along two momentum directions probed by the scattering experiment) and time. The temporal Fourier transform of such diffraction patterns results in the phonon excitation energy at each detector pixel, i.e. at each probed momentum value in the Ge Brillouin zone (the zone boundaries are indicated by the red lines). Right: Schematic illustration of how future nanoprobe femtosecond electron diffraction will enable the visualization of phonon dynamics on nanometer length scales. Shown is a focused electron beam transmitted through a sample with nanoscale inhomogeneities. The diffracted beam, indicated by the cone behind the sample, reresents a time-resolved probe of phonon dynamics similar to the one shown in the left figure panel, however, now with nanoscale resolution given by the electron beam focus.

Figure 4: A schematic time-electric field representation of an electromagnetic pulse as it can be generated using femtosecond laser and electron accelerator sources. Pulses in the $\mathrm{THz}$ frequency range can directly couple to the ferroelectric polarization in so-called multiferroic materials that are characterized by strong coupling between electrical, magnetic and lattice polarizability, indicated by the distortion (not in proportion) of a crystal lattice. The availability of such pump pulses will provide unprecedented opportunities for understanding and the ultrafast control of materials properties for use in the next-generation functional devices. Femtosecond electron pulses (not shown) will be ideally suited to image the, often inhomogeneous, sample response to such excitation fields. 